\title{
X-Ray Spectra from Neon-like Tungsten Ions in the Interaction with Electrons
}

\author{
Hirofumi WATANABE, Nobuyuki NAKAMURA, Daiji KATO ${ }^{1)}$, \\ Tomohide NAKANO ${ }^{2)}$ and Shunsuke OHTANI \\ Institute for Laser Science, The University of Electro-Communications, Chofu 182-8585, Japan \\ ${ }^{1)}$ National Institute for Fusion Science, Toki 509-5292, Japan \\ 2) Japan Atomic Energy Agency, Naka 311-0193, Japan
}

(Received 19 April 2007 / Accepted 22 May 2007)

\begin{abstract}
X-ray spectra from highly charged tungsten ions have been observed in the interaction with an electron beam at energies ranging from 14 to $1.5 \mathrm{keV}$ by using an electron beam ion trap (EBIT). In this energy range, resonant $\mathrm{x}$-ray transitions at around $9 \mathrm{keV}$ predominantly take place through dielectronic recombination processes. The measured spectra were compared with the theoretical calculation with the Hebrew University Lawrence Livermore Atomic Code (HULLAC).
\end{abstract}

(C) 2007 The Japan Society of Plasma Science and Nuclear Fusion Research

Keywords: dielectronic recombination, electron beam ion trap (EBIT), resonant process, Ne-like tungsten ion, radiative electron capture

DOI: $10.1585 /$ pfr. 2.027

In the ITER device currently being constructed in Cadarache, France, tungsten is considered to be a material of the plasma facing diverter wall due to the favorable physicochemical properties of this element [1]. For the plasma modeling, it is essential to gain a detailed understanding of the atomic processes of highly charged tungsten ions. Of all others, dielectronic recombination (DR) is the most important atomic process that plays a key role in determining the charge- and energy-balance in a hot plasma [2]. Therefore, an accurate knowledge of DR processes is needed for the development of fusion plasma research.

To date there have been few experimental investigations of DR processes of $\mathrm{W}$ ions. As the plasma temperature increases, the dominant charge state for $\mathrm{W}$ ions is considered to be changed from Ni-like to Ar-like, and to Ne-like $\mathrm{W}$ ions in a plasma with the temperature beyond several $\mathrm{keV}$, which is the most relevant species to the present fusion research. In this report, we show the x-ray spectra due to DR processes $\left(2 \mathrm{~s}^{2} 2 \mathrm{p}^{6}+\mathrm{e} \rightarrow\left(2 \mathrm{~s}^{2} 2 \mathrm{p}^{5} 3 \ln l^{\prime}\right.\right.$ or $\left.\left.2 \mathrm{~s}^{2} \mathrm{p}^{6} 3 \ln l^{\prime}\right) \rightarrow 2 \mathrm{~s}^{2} 2 \mathrm{p}^{6} 3 l+\mathrm{h} v\right)$ of highly charged $\mathrm{W}$ ions produced in the Tokyo-EBIT (Electron Beam Ion Trap), in which the Ne-like ions are abundant by controlling the electron beam energy. The Tokyo-EBIT has been partly used to provide useful atomic data for radiative and collisional processes of highly charged ions (HCIs) [3-9] in relation to fusion and astrophysical plasma research.

The EBIT consists of an electron gun, an ion trap and an electron collector. The magnetically compressed electron beam passes through the ion trap region and is collected with the collector. Ions are produced in the trap and

author's e-mail: h_watana@ils.uec.ac.jp further ionized in a stepwise fashion by electron impact until HCIs with a required charge state are produced. Radiation from the excited trapped ions is observed at $90^{\circ}$ with respect to the electron beam.

In the present study, the tungsten element is introduced into the trap as a molecular compound gas of $\mathrm{W}(\mathrm{CO})_{6}$ via a nozzle gas injection port, which immediately becomes $\mathrm{W}$ ions with high charge-states by electron impact [10]. Ne-like ions are cooked by keeping the beam energy at $14 \mathrm{keV}$ for $90 \mathrm{~ms}$. This cooking energy makes Ne-like $\mathrm{W}$ ions the most abundant in the trap, because the ionization energy of Na-like ions is $7.1 \mathrm{keV}$, while that of Ne-like ions is $14.8 \mathrm{keV}$. The electron beam energy is ramped linearly from $14 \mathrm{keV}$ to $1.5 \mathrm{keV}$, which is lower than the lowest DR resonance energy at $2 \mathrm{keV}$, and returned to the cooking energy to produce Ne-like ions again. This back and forward ramp time is fast $(10 \mathrm{~ms})$ enough to preserve the charge balance during the observation. This cooking (90 ms)-observation $(10 \mathrm{~ms})$ cycle is repeated until the clear DR X-ray spectrum can be obtained. X-ray spectra are measured with a Ge solid state detector, and recorded as a function of the electron beam energy by a list mode of the data acquisition system.

Figure 1 shows a two-dimensional map of the detected $\mathrm{x}$-ray intensity as a function of the electron energy and $\mathrm{x}$ ray energy. Several L x-rays (x-ray transition to L-shell) are seen along vertical lines at around $9 \mathrm{keV}$ which are transitions to the ground state $\left(2 \mathrm{p}^{6}\right)$ from the upper states of $\left(2 \mathrm{p}^{-1} 3 l\right)$ excited by electron impact. X-ray signals due to radiative recombination $(\mathrm{RR})$ processes are seen along diagonal lines since the RR $\mathrm{x}$-ray energy increases linearly with the electron energy. The strong $\mathrm{X}$-ray signals shown 


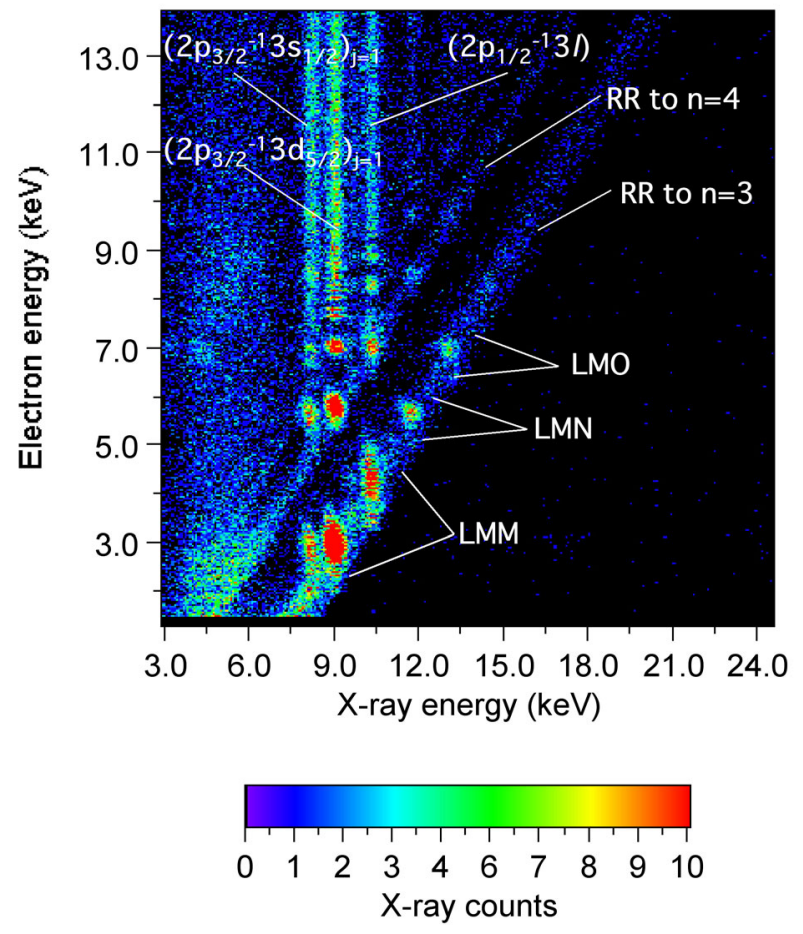

Fig. 1 Two-dimensional x-ray spectrum for Ne-like $\mathrm{W}$ ions as a function of electron energy. X-ray counts are shown to increase from black to red.

as red spots are due to $\mathrm{LM} n(n=\mathrm{M}, \mathrm{N}, \mathrm{O}, \cdots)$ DR resonances. $\mathrm{LM} n$ is the notation commonly used to specify DR resonances. For example, LMM refers to the resonant capture of a free electron into the $\mathrm{M}$ shell of an ion, at the same time exciting an $\mathrm{L}$ shell electron into the $\mathrm{M}$ shell. Since the DR processes take place competitively with the $\mathrm{RR}$ processes which have similar $\mathrm{x}$-ray energies to those of L X-ray fluorescence, the DR resonances appear at the crossings of vertical and diagonal lines.

When estimating the radiative loss rates from tungsten HCIs which exist in a hot plasma with a temperature of several $\mathrm{keV}$, it is important to know the excitation function for $\mathrm{x}$-ray emissions as a function of the electron interaction energy with a wide range around several $\mathrm{keV}$. Therefore, we derived the energy-dependent emission function of tungsten HCIs from the present observation. Figure 2 shows the relative total $x$-ray emission cross-sections as a function of the electron energy. The total emission cross-section at a given electron energy is obtained from the integration of $\mathrm{x}$-ray signals with energies ranging from $7.5 \mathrm{keV}$ to $21 \mathrm{keV}$, which contain the high energy x-ray transitions from the highly excited $n$ level. As shown in Fig. 2, in the total $\mathrm{x}$-ray emission yield spectrum, transitions due to dielectronic recombination processes (LMM, LMN, ․) have much larger contributions than the $\mathrm{L}$ x-rays $(\mathrm{L} \alpha, \beta, \cdots)$ due to direct excitation by electron impact with threshold energies at around $10.5 \mathrm{keV}$.

For Ne-like tungsten $\left(\mathrm{W}^{64+}\right)$, the DR cross-sections

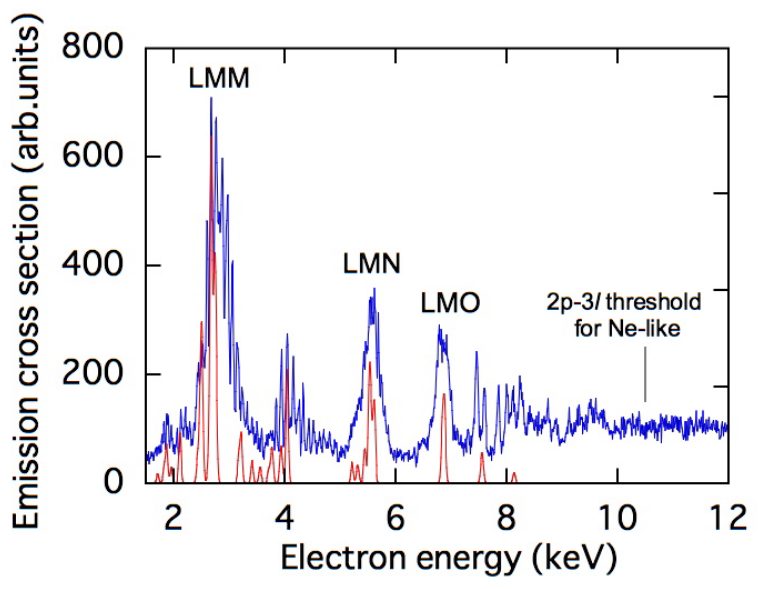

Fig. 2 Relative total $\mathrm{x}$-ray emission cross-sections for highly charged W (dominantly Ne-like) ions as a function of the electron energy. Blue and red lines are the experimental results and data from the calculation by HULLAC for Ne-like ions, respectively.

were calculated by Behar et al. [11] using the multiconfiguration relativistic Hebrew University Lawrence Livermore Atomic Code (HULLAC). In this paper, the total DR crosssections are presented in the form of $50 \mathrm{eV}$-wide peaks for individual DR resonances of $2 \mathrm{~s}^{2} 2 \mathrm{p}^{5} 3 \ln l^{\prime}$ and $2 \mathrm{~s} 2 \mathrm{p}^{6} 3 \ln l^{\prime}$ $(\mathrm{LM} n)$ states, which can be reasonably compared with the present observation, and thus shown in Fig. 2 as red lines. In comparison with the theoretical spectrum, the apparent experimental electron beam energy is shifted to the lower value by $200 \mathrm{eV}$. This shifted value is due to the potential difference of the actual electron interaction energy with trapped HCIs in the EBIT from the apparent applied voltage at the power supply, which includes the space charge potential lowering.

The experimental DR x-ray spectrum on the whole agrees well with the theoretical one. However, there are non-negligible differences which come from the contribution of x-ray emissions from lower charge-state $(q)$ ions than the Ne-like ions in the trap. When we estimate the $q$-distribution of trapped ions at the $14 \mathrm{keV}$ cooking energy and add x-ray spectra from these lower $q$ ions to that from Ne-like ions, as we have performed this treatment in the previous investigation [7], there should be better agreement.

This work was partly supported in collaboration with the Japan Atomic Energy Agency.

[1] G. Federici et al., Nucl. Fusion 41, 1967 (2001).

[2] R.K. Janev et al., Physics of Highly Charged Ions, Springer Series in Electrophysics Volume 13, ed. Guenter Ecker, (Springer-Verlag Berlin Heidelberg, 1985) p.46.

[3] H. Watanabe et al., J. Phys. Soc. Jpn 66, 3795 (1997).

[4] S. Ohtani, AIP Conf. Proc. of 8th Intl EBIS/T Conf. 572, 84 (2001). 
[5] N. Nakamura and S. Ohtani, AIP Conf. Proc. 771, 209 (2005).

[6] H. Watanabe et al., J. Phys B: At. Mol. Opt. Phys. 34, 5095 (2001).

[7] Y.M. Li et al., J. Plasma, Fusion Res. 77, 1175 (2001).
[8] B. O'Rourke et al., J. Phys B: At. Mol. Opt. Phys. 37, 2343 (2004).

[9] H. Watanabe et al., Phys. Rev. A 75, 012702 (2007).

[10] C. Yamada et al., J. Phys: Conf. Ser. 58, 403 (2007).

[11] E. Behar et al., Phys. Rev. A 59, 2787 (1999). 